\title{
Téoros
}

Revue de recherche en tourisme

\section{Les sciences du tourisme en quête de légitimité}

\section{Gilles Pronovost}

Volume 27, numéro 1, printemps 2008

Science du tourisme ou études touristiques?

URI : https://id.erudit.org/iderudit/1070892ar

DOI : https://doi.org/10.7202/1070892ar

Aller au sommaire du numéro

Éditeur(s)

Université du Québec à Montréal

ISSN

0712-8657 (imprimé)

1923-2705 (numérique)

Découvrir la revue

Citer cet article

Pronovost, G. (2008). Les sciences du tourisme en quête de légitimité. Téoros, 27(1), 18-21. https://doi.org/10.7202/1070892ar d'utilisation que vous pouvez consulter en ligne.

https://apropos.erudit.org/fr/usagers/politique-dutilisation/ 


\section{Les sciences du tourisme en quête de légitimité}

\section{Gilles Pronovost}

On répète souvent que les études du tourisme sont récentes. Le tourisme lui-même est certes plus ancien, mais il est presque dans la "nature " des sciences sociales de se pencher sur des «problèmes » bien longtemps après que ceux-ci soient apparus. Ainsi ce n'est que dans la deuxième moitié du siècle dernier que des chercheurs se sont penchés sérieusement sur l'histoire du tourisme... que l'on fait pourtant remonter au XVIIII siècle, voire avant. De même, dans le cas du loisir contemporain, il faut attendre le premier tiers du siècle dernier pour repérer des chercheurs en sciences sociales qui s'intéressent à cette question, tout particulièrement en philosophie et en anthropologie. S'il faut qu'un «problème » surgisse pour que l'on en vienne à s'y intéresser en sciences sociales, même avec un décalage de plusieurs décennies, les traditions disciplinaires existantes ont parfois beaucoup de poids dans les premiers repérages que l'on tente d'en faire.

Notre propos ici est de décrire brièvement les premiers moments de cette histoire des sciences sociales du tourisme, soit par extension de notions déjà existantes, soit par l'appel à des concepts classiques que l'on adapte au phénomène en question, soit par la recherche de marqueurs plus ou moins spécifiques, pouvant éventuellement servir d'argument au caractère " distinct » du tourisme par rapport à tout autre champ.

\section{Entre loisir et tourisme}

Le cas le plus typique est celui de l'extension dans un champ présumé nouveau de notions tirées de savoirs spécialisés déjà constitués. À cet égard, dans le cas du tourisme, les emprunts aux sciences du loisir sont notoires. Marc Boyer par exemple, dans Le tourisme, ouvrage classique publié en 1972 et réédité une dizaine d'années plus tard, commence par définir... non pas le tourisme, mais bien le loisir. Tourisme et loisir se voient accordées les mêmes fonctions; plus encore, le phénomène touristique est abordé selon le même cadre de référence, tel qu'emprunté aux catégories classiques de Joffre Dumazedier (présentées dans son ouvrage de 1966) sur les intérêts culturels. On peut dire que, dans ce cas, le phénomène plus général semble le loisir, dont le tourisme semblerait une ex-croissance pour satisfaire un besoin culturel de civilisation industrielle (ce sont les termes mêmes de Boyer). Comment ce «besoin » est-il comblé ? En se déplaçant de chez soi à ailleurs. On verra plus loin que les géographes se sont emparés de cette notion de déplacement pour tenter d'y situer le point focal de la spécificité du tourisme contemporain.

Les travaux de Dean MacCannel (1976) empruntent également la voie de la sociologie du loisir pour tenter de cerner le phénomène touristique. D'entrée de jeu, celui-ci soutient que le développement des sociétés modernes doit être rapporté au développement des loisirs de masse, dont le tourisme international et le sightseeing constituent la figure principale. Chez lui, loisir et tourisme sont si intimement liés qu'il n'hésite pas à écrire que son ouvrage est d'abord un essai non pas de sociologie du tourisme, mais bien de sociologie du loisir. À juste titre, on retient surtout de cet auteur ses remarques pénétrantes sur le « voyeurisme touristique », les étapes de la "sacralisation touristique", la construction sociale des attractions touristiques, mais on oublie un peu vite leur cadre de référence emprunté à la sociologie du loisir.
Dans la même veine, les travaux plus récents de Chris Rojek et John Urry (1997) se veulent une perspective critique sur le tourisme, à partir des cadres de référence de la sociologie du loisir et de la culture. S'inspirant de D. MacCannel, ils mettent l'accent sur les frontières difficiles à tracer entre loisir, société de consommation et tourisme, traitent de la figure du tourisme contemporain en termes de modernité et de postmodernité, parlent des touristes comme acteurs de leur propre performance.

\section{Géographie du tourisme ou du loisir? La quête des fondements}

Cet amalgame plus ou moins reconnu entre les deux phénomènes - loisir, d'une part, tourisme, d'autre part - est vite devenu insupportable pour les tenants d'une science sociale à part entière, autonome, libérée des cadres de la sociologie du loisir. Car, dans cette perspective, le tourisme apparaissait comme un phénomène dérivé du loisir, finalement de moindre dimension, sans différence fondamentale apparente. La recherche de la spécificité du tourisme ne s'est pas faite sans mal. II n'a en effet pas été facile de s'émanciper du cadre de référence de la sociologie du loisir. II fallait tenter de constituer le phénomène touristique en un champ scientifique autonome. Prenons pour illustration les travaux de Gabriel Wackerman, géographe bien connu. N'a-t-il pas publié, deux décennies après l'ouvrage pionnier de Marc Boyer, un livre important débutant pourtant ainsi : Loisir et tourisme... ? Ce n'est qu'en deuxième lieu qu'il ajoute: Une internationalisation de l'espace (1994). Signe évident de l'amalgame entre les deux phénomènes, il écrit : "le loisir est à la base du tourisme. II constitue, au moins en principe, l'élément apparent le plus important de celui-ci [...] Ainsi, le tourisme, 'dérivé' du loisir ou plutôt 'quête élargie du loisir' est inté- 
gré de façon croissante à l'économie générale tout court et donne-t-il lieu au déploiement d'une véritable économie du loisir. " (p. 9-10) II évoque même la finalité réciproque de l'un et l'autre phénomène. Presque une décennie plus tard, à ma demande, il reviendra sur ces distinctions entre loisir et tourisme pour préciser : "Le tourisme est un acte dérivé du loisir ou une quête élargie sur le plan spatial. II consiste, à vrai dire, en un loisir lié à un déplacement en vue d'un certain dépaysement. » (1998: 484)

Ce sont les notions de "déplacement " et "d'espace », puis de «territoire », qui sont venues à la rescousse des tenants de la spécificité du tourisme par rapport à tout autre phénomène. Marc Boyer y avait déjà fait allusion, les géographes s'en s'ont emparés, délaissant peu à peu les cadres sociologiques pour des approches nettement influencées par la discipline de la géographie. Dans Tourisme et transport (1993), G. Wackerman n'écrit-il pas : "Par sa nature même, le tourisme implique le transport, puisqu'il exprime le loisir en déplacement » (p.60) ? Ainsi, il suffit de déplacer les termes : le loisir chez cet auteur est progressivement relié à la notion de sédentarité (on sait les connotations péjoratives qu'elle implique), le tourisme à la mobilité dans le temps et l'espace.

Reste un pas à franchir : tenter de définir l'essence même du tourisme. Si l'on demeure dans cette comparaison entre la constitution des sciences du loisir et celle du tourisme, on se rappellera que les travaux pionniers sur le loisir se sont lancés dans une quête éperdue de son essence... que l'on a cru trouver dans des notions de « liberté ", sinon d'état d'esprit (chez Neulinger, 1980 notamment). Nous avons illustré ailleurs que la pensée américaine dominante sur le loisir du début du $X X^{e}$ siècle était profondément ancrée dans une certaine théorie du jeu, de la nature humaine et de la civilisation (Pronovost, 1983). Les sciences du loisir ont longtemps vécu sur l'illusion qu'un jour elles en atteindraient l'essence, ou encore qu'elles pourraient s'appuyer sur le socle d'une définition de la nature humaine.

De manière analogue, les travaux pionniers sur le tourisme n'ont pu résister à cette quête de ses fondements ultimes. Revenons à l'exemple des travaux de G. Wackerman. Les fondements de son approche (celle du déplacement associé à la notion même de

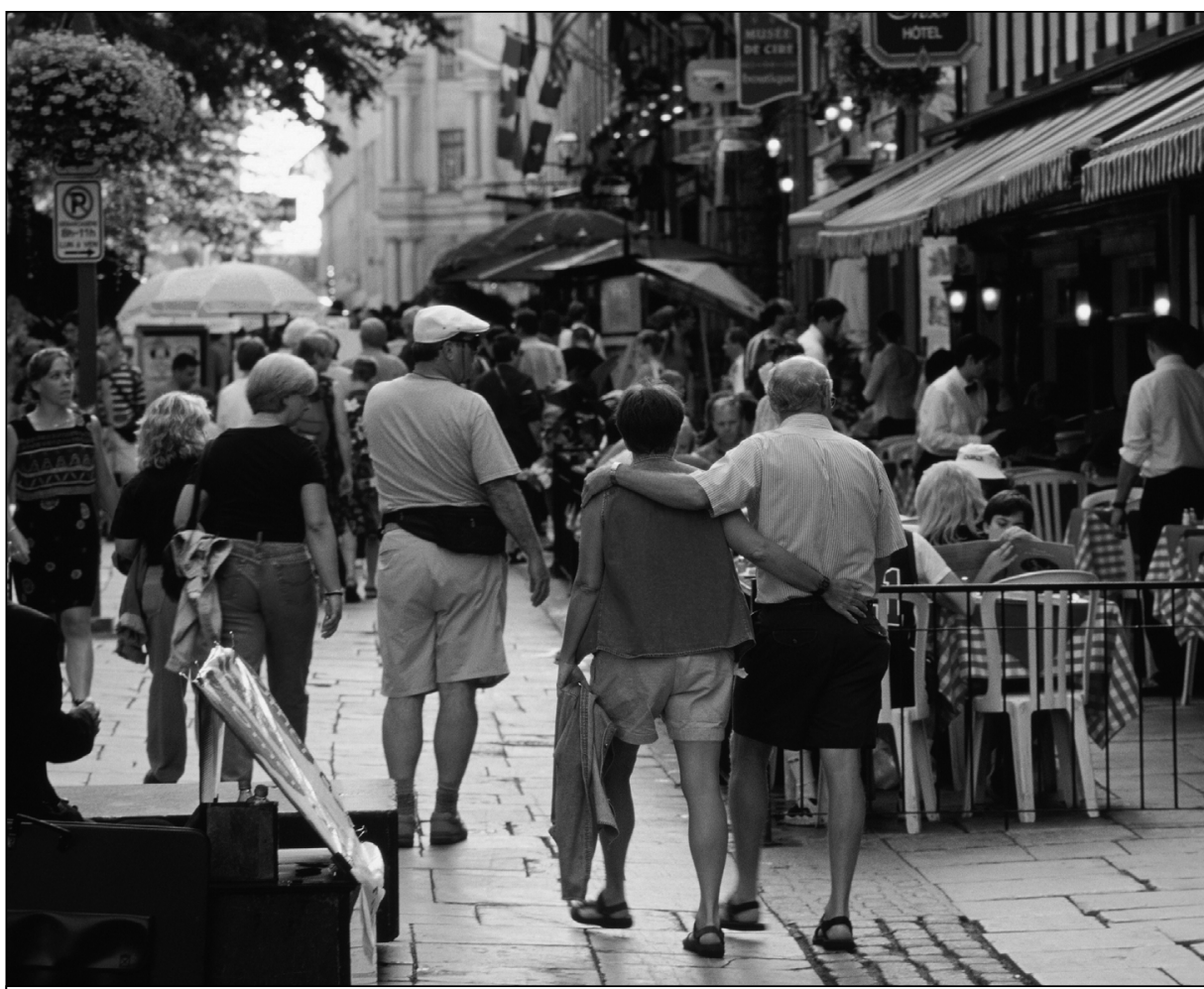

Terrasse, rue Sainte-Anne, Vieux-Québec.

Photo : Linda Turgeon

tourisme) s'orienteront naturellement dans une recherche de ce qui peut ainsi pousser l'homme à vouloir voyager et se déplacer. La tentation d'une certaine définition de l'homme «naturel » est tout proche : celui-ci aurait une propension innée à voyager, il aurait naturellement soif de connaître, soif de découvrir. Selon cet auteur, "L'essence même du loisir et du tourisme réside dans cette tendance universelle à alimenter l'imagination à la fois par des faits vérifiés et des représentations en provenance de toutes sortes de cadres de référence: religion, idéologies, philosophies, éducation d'origine diverse, vécu, formation. » (1994: 17) Par ailleurs, "Loisir et tourisme ne sont pas idéologiquement neutres ni innocents, ils relèvent d'une optique, d'un engagement, d'une conception sociale, de la dignité humaine. » (1994: 38)

On peut y voir ici une relation typique entre la «production de concepts » et leur origine disciplinaire. Pour s'affranchir d'une discipline, il suffit de faire appel à une autre. Plus encore, on peut y déceler une tentative subtile, après avoir reconnu la primauté, l'antériorité, du loisir par rapport au tourisme, d'établir progressivement une autre hiérarchie, qui fait cette fois du tourisme un phénomène pré- senté comme plus large, plus important, sinon premier. C'est la voie royale qu'empruntent souvent les chercheurs en sciences sociales: à savoir, des tentatives de légitimation de leur champ (en l'occurrence le tourisme), présenté comme "spécifique » ou encore «plus global » qu'un autre champ qui sert de référence (généralement celui du loisir).

II ne reste qu'à faire appel à certains attributs "innés » de la "nature humaine » pour conclure.

Les notions de déplacement et d'espace, tout particulièrement, constituent une tentative plus ou moins explicite de délimiter un champ scientifique à conquérir. À notre connaissance, c'est la discipline de la géographie qui est allée le plus loin dans cette tentative de définir la spécificité du tourisme, essentiellement par rapport au loisir qui en est presque venu à lui servir de repoussoir. Un peu comme dans le cas des études du loisir, qui ont longtemps tenté d'en définir la spécificité, la nature intrinsèque, le caractère irréductible, la géographie s'est lancée de la même manière à la conquête du champ du tourisme, clamant haut et fort ses traits irréductibles. 
D'autres chercheurs ont aussi cherché le graal du tourisme, proposant un ou deux aspects présentés comme spécifiques. On peut donner l'exemple de J. Urry (2002), qui voit dans le tourist gaze, un trait qui s'approche de l'essence du tourisme. L'homo touristicus serait un être de curiosité sans fin, éperdument en quête d'ailleurs renouvelés, même au prix des constructions artificielles à la Disneyland. Chez J. Urry, cependant, le tourisme en vient progressivement à se dissoudre dans des caractéristiques des sociétés contemporaines et on ne voit plus bien, aux termes de sa démarche, ce qu'il en reste vraiment. Rappelant que les sociétés occidentales tout au moins ont changé rapidement depuis les dernières décennies, il en conclut que la nature du tourisme et de la curiosité touristique a changé également. «Ce que j'appelle le tourist gaze devient de plus en plus relié à toutes sortes d'autres pratiques sociales et culturelles et s'en distingue de moins en moins " (édition de 1990 : 82, traduction libre). La conséquence est le déclin de la spécificité du tourisme en tant que tel, on dirait aujourd'hui la mondialisation de la curiosité touristique. II en voit des exemples dans la prédominance de la « forme romantique " du tourisme, de la recherche du « naturel », de «l'authenticité », du développement du tourisme rural, de l'écotourisme pourrait-on ajouter. Dans la même veine, C. Rojek parle du sight framing (1997), qui est devenu un aspect intrinsèquement lié à la culture contemporaine.

Nous savons bien qu'il s'agit d'une voie sans issue. Les sujets qui se portent à l'attention des chercheurs varient beaucoup dans le temps. Les thématiques du travail, de la famille et de la culture en constituent des exemples éloquents. Ces perspectives changeantes rendent d'ailleurs la recherche vulnérable aux conjonctures économiques, politiques ou sociales d'une époque. Max Weber (1965) a depuis longtemps décrit comment les "intérêts de connaissance ", comme source de questionnement des sciences sociales, sont fonction de l'histoire. II y a une historicité inhérente au questionnement des sciences sociales et l'on ne voit pas comment les sciences sociales du tourisme y échapperaient.

Par exemple, dans un article bien étoffé, Graham Dann (2000) illustre ces perspectives changeantes. II décrit comment les études touristiques seraient passées d'approches essentiellement descriptives à l'étude des motivations, de l'accent mis sur les relations hôtes-visiteurs aux questions d'altérité et de rapports hommes-femmes, et ainsi de suite. Tel que nous le rappelons plus haut, C. Rojek et J. Urry (1997) proposent la notion de «post-modernisme » pour décrire certains traits actuels du touriste. Et c'est sans compter la vogue actuelle des études en tourisme culturel.

Bref, il n'y a pas et il ne peut y avoir de point focal dans quelle que discipline des sciences sociales que ce soit. Nous ne savons jamais tout à fait si l'angle de questionnement que nous privilégions est tributaire de notions courantes héritées de la culture et des modes de vie qui nous façonnent en partie, s'il n'est pas biaisé par les questions à la mode, s'il ne s'avère pas profondément «occidento-centriste » dans ses fondements.

\section{Sciences sociales de tout et de rien ? La fragmentation du savoir}

L'entrée en scène de diverses disciplines classiques marque une nouvelle étape dans l'histoire des sciences sociales du tourisme. Le cas qui nous semble le plus typique est celui de la psychologie, même si les travaux ne sont pas légion. En généralisant beaucoup on peut dire qu'à ce jour les tenants de cette discipline se sont surtout bornés à reprendre les grands concepts classiques de la psychologie, extensionnés à l'étude du touriste. Ainsi, Chris Ryan (1991) développe une psychologie axée sur «l'expérience touristique ", qui connaîtra, on le sait une certaine faveur. Les notions de motivation, de satisfaction, sont également utilisées. La spécificité tient ici non pas à des caractéristiques inhérentes au phénomène étudié, mais au caractère disciplinaire des concepts et des théories utilisés.

De même, les brèves incursions des économistes s'en tiennent souvent à des approches classiques: insistance sur les aspects économiques, contribution du tourisme au développement régional, dimensions internationales. Elles s'articulent encore autour des notions d'offre et de demande, d'impacts, de comptes économiques (Raboteur, 2000). Beaucoup d'aspects techniques caractérisent cette approche; C. Rojek et J. Urry ne manquent pas d'en dénoncer la positivist operationalisation ainsi que ses traits réducteurs. Dans un certain sens, on peut encore souligner que les études économiques ont grandement servi à la justification de « l'importance » du tourisme et du trop peu de cas qu'on en fait en sciences sociales.

Par ailleurs, comme on l'a démontré à quelques reprises, le paysage scientifique du tourisme a non seulement beaucoup évolué, au gré des questionnements scientifiques, historiques, politiques et sociaux, mais il a aussi éclaté. La recherche de François Bédard et Boualem Kadri (2001) est révélatrice à cet égard. On y lit notamment, en filigrane, la montée de thématiques industrielles, managériales et économiques. Ces auteurs se demandent - encore - si une science du tourisme est toujours possible, soulignant explicitement qu'ils entendent par là " une science du tourisme constituée et autonome " dont ils déplorent la faible reconnaissance sociale. II est significatif qu'ils appellent à la rescousse l'économie et la psychologie, présentées comme étant en mesure de fournir les outils théoriques et méthodologiques nécessaires.

On peut ainsi constater que si la sociologie et l'anthropologie, avec l'histoire, ont offert les premiers constituants d'une science sociale du tourisme, et demeurent de nos jours pratiquement les seules approches véritablement critiques, c'est bien la géographie qui a investi de manière significative ce champ et qui a tenté d'en définir la spécificité. L'économie et la psychologie sont intervenues par la suite, suivies de travaux de nature opérationnelle (gestion, management, etc.). Elles semblent servir de repères pour l'ancrage méthodologique des études en tourisme. On décèle encore les traces d'un discours déplorant le faible niveau de reconnaissance de cette discipline. Les thématiques du déplacement et du dépaysement restent encore très fortes, la tentation de «l'homme naturel » et de la « nature au naturel » demeure encore prégnante.

II en résulte une réelle fragmentation du savoir, en vertu de laquelle querelles disciplinaires et querelles de frontières ne sont pas absentes. Mais ce n'est pas le propre des sciences sociales du tourisme; le domaine qui nous est le plus familier, le loisir, est aussi aux prises avec la même problématique.

Face à cette situation, une autre tentation guette les chercheurs: celle du holisme. Les sciences du loisir ont connu cet épisode : en « rapiéçant » diverses notions et disciplines, en cherchant désespérément une 
sorte d'unicité à leur champ de recherche, on pensait enfin parvenir à une synthèse des grandes thématiques aux fondements du loisir moderne. On peut repérer la même tentation dans certains travaux actuels du domaine du tourisme. Tel nous semble le cas du traité de tourismologie de Jean-Michel Hoerner (2002): on peut y lire un tableau dans lequel sont mis en parallèle la sociologie, la géographie et l'économie, la « tourismologie " étant présentée bien entendu comme l'ultime voie (Bédard et Kadri, dans leur article déjà cité, appellent également à «la construction de paradigmes»).

\section{Conclusion}

Ce tour d'horizon est forcément trop rapide. Les raccourcis n'échapperont pas au lecteur, mais il nous a semblé qu'il peut servir au développement d'une perspective critique des sciences sociales du tourisme, qui tarde encore trop à venir. Que l'on cherche à légitimer son champ de recherche, rien de plus naturel. II suffit de faire appel à la notion de «problème social » nécessitant un questionnement, des cadres d'analyse et des méthodologies empruntés aux sciences sociales. Une telle démarche est différente de celle qui consiste à chercher des "fondements ", à " conquérir » un champ, voire à se l'approprier. Elle s'oppose même à toute construction fondée sur une quelconque définition de l'essence ou de la nature ultime de quel que phénomène que ce soit. Elle rappelle le caractère changeant et historique des thématiques de recherche.

Les sciences sociales du tourisme ont-elles un avenir? Pour répondre à cette question, très partiellement, nous proposons quelques réflexions issues de notre propre expérience de recherche dans le domaine des sciences du loisir. En premier lieu, l'histoire des sciences du loisir témoigne qu'il est vain de vouloir chercher quelque fondement ultime, quelque paradigme, à tout champ de recherche. II n'existe aucun point de référence définitif sur lequel appuyer la recherche scientifique en sciences sociales, puisque l'on a affaire à des phénomènes qui sont, en raison de leur nature même, changeants, culturellement, historiquement et idéologiquement chargés.

De même, nous devons écarter la vaine quête d'une approche holistique; si nous nous fions à notre expérience des sciences du loisir, cette démarche a essentiellement abouti à des propos superficiels, abusivement présentés comme synthétiques, donnant l'illusion d'une compréhension globale, parfois proche de la tautologie.

Enfin, les sciences sociales du tourisme font l'expérience de la compétition entre diverses disciplines pour s'accaparer de ce champ, pour le légitimer à leur manière, sinon pour imposer leur position dominante. Ces tentatives sont vouées à l'échec. La fragmentation du savoir est au bout de chemin. II n'y a ici d'autres voies que de s'ouvrir au dialogue interdisciplinaire, au prix du renoncement à certaines notions que l'on croit fondées.

De plus, les études en tourisme pourraient faire montre d'un esprit plus critique sur certaines notions idéologiquement et culturellement marquées: n'y a-t-il pas des conceptions un peu trop idéologiques de la nature, de l'authenticité, du développement régional, du tourisme dit culturel, voire de l'écotourisme, dans certains travaux, dont le contenu résisterait peu à une analyse anthropologique critique?

Gilles Pronovost est professeur associé au Département des études en loisir, culture et tourisme de l'Université du Québec à Trois-Rivières.

\section{Bibliographie}

«Le tourisme » (1988), Loisir et Société / Society and Leisure, vol. 11, nº 1.

Bédard, François et Boualem Kadri (2001), « La recherche en tourisme en Amérique du Nord francophone et anglosaxone à travers quelques revues. États des lieux (19701999) ", La recherche en tourisme. Actes du Colloque de Foix, Paris, LT Éditions Jacques Lanore, p. 67-76.

Boyer, Marc (1972), Le tourisme, Paris, Seuil.

Boyer, Marc (1997), «Le tourisme: une épistémologie spécifique en quête de paradigmes ", Loisir et Société / Society and Leisure, vol. 20, n 2, p. 455-477.

Dann, Graham (2000), « Theoretical Advances in the Sociological Treatment of Tourism ", dans Stella R. Quah et Arnaud Sales (dir.), The International Handbook of Sociology, London, Sage, p. 367-384.

Dumazedier, Joffre et Aline Riper (1966), Loisir et culture, Paris, Seuil, $398 \mathrm{p}$.

Hoerner, Jean-Michel (2002), Traité de tourismologie. Pour une nouvelle science touristique, Perpignan, Presses universitaires de Perpignan.
Holden, Andrew (2005), Tourism Studies and the Social Sciences, London, Routledge.

Holloway, J. Christopher (2006 [7e éd.]), The Business of Tourism, Atlanta, Prentice Hall.

Jafari, Jafar, (1999), The Scientification of Tourism, University of Wisconsin.

MacCannell, Dean (1976), The Tourist. A New Theory of the Leisure Class, New York, Schocken Books.

Neulinger, John (1980), To Leisure: An Introduction, Boston, Allyn and Bacon.

Pronovost, Gilles (1983), Temps, culture et société, Québec, Presses de l'Université du Québec.

Pronovost, Gilles (1997), «Les études du loisir : héritage et perspectives", Loisir et Société/ Society and Leisure, vol. 20, n 2, p 349-367.

Raboteur, Joël (2000), Introduction à l'économie du tourisme, Paris, L'Harmattan.

Ryan, Chris (1991), Recreational Tourism: A Social Science Perspective, London, Routledge.

Ryan, Chris, (dir.) (1997), The Tourist Experience. A New Introduction, London, Cassel.

Rojek, Chris et John Urry, (dir.) (1997), Touring Cultures. Transformations of Travel and Identity, London, Routledge.

Ross, Glenn F. (1998) [1994], The Psychology of Tourism, Melbourne (Australie), Hospitality Press.

Smith, Melanie K. (2003), Issues in Cultural Tourism Studies, London, Routledge.

Stafford, Jean (2003), « La crise de la recherche en tourisme: la solution est dans les problèmes ", Loisir et Société / Society and Leisure, vol. 26, n० 1, p. 87-95.

Urry, John, (1990), The Tourist Gaze. Leisure and Travel in Contemporary Societies, London, Sage, $192 \mathrm{p}$.

Urry, John (2002 [2e éd.]), The Tourist Gaze. Leisure and Travel in Contemporary Societies, London, Sage.

Vander Gucht, Daniel (2006), Ecce homo touristicus. Identité, mémoire et patrimoine à l'ère de la muséalisation du monde, Lovernal, Éditions Labor.

Wackermann, Gabriel (1993), Tourisme et transport, Paris, SEDES.

Wackermann, Gabriel (1994), Loisir et tourisme. Une internationalisation de l'espace, Paris, SEDES.

Wackermann, Gabriel (1998), "Loisir et tourisme dans les mutations de la société contemporaine : réflexions sur un bilan scientifique", Loisir et Société / Society and Leisure, vol. 20, no 2, p. 479-501.

Wackermann, Gabriel (2007), "Tourisme, mondialisation et risques territoriaux ", Loisir et Société / Society and Leisure, vol. 30, no 1 , p. 197-219.

Weber, Max, (1965), Essai sur la théorie de la science, Paris, Plon. 